\title{
Is There Any Link Between Cardiovascular Disease, Obesity And Periodontal Disease?
}

\author{
${ }^{1}$ Dr.Preethe Paddmanabhan*
}

\begin{abstract}
Many people in the world are affected by hyperlipidemia, which is a known risk factor for atherosclerotic disease. On the other hand periodontitis, a prevalent oral disease has been connected to several systemic health changes, including an altered lipid metabolism. Obesity is a risk factor for several chronic diseases most notably hypertension, type II diabetes, dyslipidemia and coronary heart disease. Obesity may have a deleterious consequences on the the host response, leading to alternations in $T$ cell and monocyte, macrophages, increased cytokine IL-1 and IL-6 and tumor necrosis factor alpha. These observations suggest a potential interaction among obesity, periodontitis and chronic disease incidence. Thus in addition to being a risk factor for type 2 diabetes and coronary heart disease, obesity related inflammation may also promote periodontitis. Conversely periodontitis, once existing may promote systemic inflammation, thereby increasing the risk of coronary heart disease.

Key words: Hyperlipidemia, atherosclerotic disease, Obesity, Periodontitis,
\end{abstract}

\section{Introduction}

Periodontal disease is multifactorial in etiology. In the recent years it has been recognized that several systemic conditions appear to be associated with patients who have periodontal disease. Chronic Periodontitis which is the most frequent form has been defined as an infectious disease resulting in the inflammation within the supporting tissues of the teeth, progressive attachment loss, and bone loss ${ }^{1}$. The chronic destruction of these supporting tissues leads to the eventual loss of teeth and hence partial or complete loss of teeth.

Many systemic diseases and disorders have been implicated as risk factors in periodontal disease. These factors include cigarette smoking; systemic diseases such as diabetes mellitus, medications like steroids, anti-epilepsy drugs and cancer therapy drugs; ill-fitting bridges; crooked teeth and loose fillings; pregnancy; and oral contraceptive use. In addition to these variables, any medical condition that triggers host antibacterial defense mechanisms, such as human immunodeficiency virus (HIV) infection, diabetes, and neutrophil disorders, will likely promote periodontal disease. Chronic bacterial infection including periodontitis have been associated with the increase risk of atherosclerosis and coronary heart disease ${ }^{2}$.

\section{Obesity - Definition and assessment:}

Obesity, defined as a body mass index (BMI) $\geq 30.0 \mathrm{~kg} / \mathrm{m} 2$, is a major public health problem today . The definition of obesity is based on body mass index (BMI, also called Quetelet Index), which is the ratio of body weight (in $\mathrm{kg}$ ) to body height (in $\mathrm{m}$ ) squared ${ }^{3}$. Obesity is a risk factor most or for several chronic diseases most notably hypertension, type II diabetes, dyslipidemia and coronary heart disease further, recent studies have suggested that obesity is also related to oral diseases particularly with periodontitis ${ }^{4,5}$

Obesity is characterized by the abnormal or excessive deposition of fat in the adipose tissue. Its consequences go far beyond adverse metabolic effects on health, causing an increase in oxidative stress, which leads not only to endothelial dysfunction but also to negative effects in relation to periodontitis, because of the increase in proinflammatory cytokines.

BMI is highly correlated with fat mass and morbidity and mortality and therefore sufficiently reflects obesity-related disease risk in a wide range of populations; however, there are some limitations. For example, for the same BMI, older persons tend to have a higher body fat composition; and therefore, risk assessment by BMI is less accurate in older people (over 65 years of age). Body fat distribution is assessed by the measurement of waist circumference, with $102 \mathrm{~cm}$ in men and $88 \mathrm{~cm}$ in women, respectively, being the cutoff point for abdominal obesity associated with an increased risk of morbidity ${ }^{3}$.

Waist circumference shows a close correlation with the amount of visceral adipose tissue, and visceral adipose tissue has been shown to be metabolically more active and to secrete far greater amounts of cytokines and hormones compared with subcutaneous adipose tissue. ${ }^{(6)}$ Recently, numerous studies have indicated that measurement of the waist circumference or waist-hip ratio may be a better disease risk predictor than BMI, and there is still intensive research ongoing as to whether BMI, waist circumference or both should be used to assess disease risk ${ }^{7,8}$. 


\begin{abstract}
II. Pathophysiology
Relationship between Periodontitis, Obesity \& Chronic inflammation

Several studies have indicated that subjects with periodontal disease may have a higher risk for cardiovascular disease when compared to subjects with a healthy periodontium. It has been suggested that obesity is second only to smoking as the strongest risk factor for inflammatory periodontal tissue destruction ${ }^{11}$.Periodontitis- induced changes in immune cell function may cause metabolic dysregulation of lipid metabolism through a mechanism involving proinflammatory cytokines ${ }^{17}$. Systemic exposure to infectious challenges such as bacterial lipopolysaccharide, as in periodontitis, can result in the release of inflammatory cytokines such as IL-1 $\beta$ and Tumour Necrosis Factor alpha (TNF $-\alpha)$ that alter fat metabolism and promote hyperlipidemia ${ }^{17}$.
\end{abstract}

\title{
Adipose tissue derived cytokines and Harmones
}

Interleukin-6: Interleukin-6 is secreted by the human adipose tissue and is produced in greater amounts by deep abdominal fat than by subcutaneous fat. Elevated levels of interleukin- 6 have been found to be associated with increased risk of cardiovascular events, lipolysis and weight gain ${ }^{9}$.

TNF- $\square$ : Obesity-associated tumor necrosis factor- $\alpha$ (TNF- $\alpha$ ) is primarily secreted from macrophages accumulated in the abdominal adipose tissue. Increased circulating TNF- $\alpha$ from the adipose tissue contributes to poor health outcomes by increasing insulin resistance, C-reactive peptide production and general systemic inflammation. TNF- $\alpha$ is a potent inhibitor of adiponectin, an important anti-inflammatory adipokine ${ }^{9}$.

Adiponectin: Adiponectin is a circulating hormone secreted by the adipose tissue. It is involved in glucose and lipid metabolism and accounts for about $0.05 \%$ of the total serum proteins. Contrary to other adipose-derived hormones, adiponectin levels are reduced in persons with obesity, insulin resistance or Type 2 diabetes.

Leptin: Leptin is the best known substance secreted from the adipose tissue. It plays an important role in regulating energy intake and energy expenditure, including appetite and metabolism.

Resistin: Resistin belongs to a family of resistin-like molecules (RELM) and has been reported to be secreted by adipocytes and to cause insulin resistance in animal models. Current evidence suggests that in humans, resistin is more closely related to inflammatory processes than to insulin resistance. Whether or not resistin plays a role in inflammatory periodontal disease remains to be defined ${ }^{10}$. Plasminogen activator inhibitors-1 (PAI-1): PAI-1 is an adipokine which generates agglutination of blood and raises the risk of ischemic vascular disease and gingival inflammation. PAI-1 may decrease blood flow in the periodontium of obese patients and promotes development of periodontitis ${ }^{6}$.

REACTIVE OXYGEN SPECIES: It is also believed that there is a close association of obesity and periodontitis with chronic inflammation. Reactive oxygen species are the products of normal cellular metabolism, but over production of reactive oxygen species, induces damage by oxidizing DNA, lipids and proteins. Obesity increases the circulation of reactive oxygen species which in turn causes gingival oxidative damage and progression of periodontitis.

Inflammatory diseases like periodontitis induce pro inflammatory cytokines such as TNF- $\alpha$, IL-1 and IL- $6^{14}$ It has been suggested that the secretion of TNF- $\alpha$ by adipose tissue triggered by LPS from gram negative bacteria promote hepatic dyslipidemia and decrease insulin sensitivity ${ }^{15}$. Type 2 diabetes and decrease insulin sensitivity are associated with the production of advanced glycation end-products (AGE) which trigger inflammatory cytokine production, thus pre disposing for inflammatory disease such as periodontitis ${ }^{16}$.

Genco et al suggested that obesity was associated with periodontal infection through inflammation. Lipids (eg fatty acids, cholesterol, or fat soluble vitamins) and glucose can also deeply affect the immune system. In addition modulation of immune responses via interactions with toll like receptors at the surface of immune cells, lipids seem to be essential for the performance of immune cells as energy supplier and as constituents of the membrane architecture. Generating an efficient and effective immune response involves large increases in cellular proliferative biosynthetic and secretory activities, which require high energy consumption. Therefore, innate immune cells must rapidly respond to the presence of pathogens shifting from a quiescent phenotype to a highly active state within hrs after stimulation ${ }^{13}$.

Thus obesity appears to participate in the multifactorial phenomenon of causality of periodontitis through the increased production of reactive oxygen species. The possible causal relationship between obesity and periodontitis and potential underlying biological mechanisms remain to be established; however, the adipose tissue actively secretes a variety of cytokines and hormones that are involved in inflammatory processes, pointing toward similar pathways involved in the pathophysiology of obesity, periodontitis and related inflammatory diseases. 


\section{Link between cardiovascular disease and periodontal disease}

The majority of risk factors for CVDs are also considered to be risk factors for periodontal diseases. The presumed association between the poor oral hygiene and atherosclerosis can be explained by the effect of chronic inflammatory disease on blood components and direct effects of active cytokines and activated lymphocytes and monocytes on vascular tissue A hypothetical relationship between damage to periodontium and atherosclerosis could be explained by the effect of a chronic inflammatory process on blood rheology, and a direct effect of active cytokines and activated lymphocytes and monocytes on the tissues of blood vessel walls. Periodontitis is a chronic inflammatory disease that is characterized by a Th1 reaction and the release of cytokines (such a TNF- $\alpha$ and IL- $\beta$ ). It is a well established fact that these cytokines also influence the development and progression of atherosclerosis

Proinflammatory cytokines may play a crucial role in the relationship between periodontitis, obesity, and chronic diseases ${ }^{17}$. In fact this association may be multidirectional. For example, it has been well established that inflammation is an essential component in the development of atherosclerosis and observational studies showed that periodontitis is associated with a moderately, but significant higher risk of coronary heart disease ${ }^{17}$.

\section{Role of General physician and dentist in management of obesity and periodontal disease}

1. Weight loss therapy is recommended for patients with a body mass index of $\$ 30$ and for patients with a body mass index of 25-29.9, or a high-risk waist circumference, and two or more risk factors. Faster rates of weight loss are no more effective over the long term.

2. Primordial prevention of diabetes includes targeting the prenatal state, childhood, and adolescence to promote healthy lifestyles before risk factors are acquired. Today, it is known that the atherosclerotic process begins in youth, culminating in the risk factor-related development of vascular plaque in the third and fourth decades of life. Good nutrition, a physically active lifestyle, and absence of tobacco use contribute to lower risk prevalence and either delay or prevent the onset of cardiovascular disease ${ }^{12}$.

\section{Conclusion}

Obesity is a complex disease, and its relationship to oral status has been realized by the scientific community in recent years. Obesity is a complex and multifactorial disease. Its relationship with periodontal disease and other chronic disease is well documented but underlying mechanism is under investigation. Chronic Inflammation has multidirectional link with periodontal disease, obesity and other chronic conditions. A periodontist can educate his patients about related information and can help in improving oral and overall health of patient.

[1] Flemming TF: Periodontitis, Ann Periodontol 4:32,1999.

[2] Mattila KJ, Valtonen VV et al. Role of infection as a risk factors atherosclerosis, myocardial infarction and stroke. Clin Infec Dis. 1998;26:719-734.

[3] Expert Panel on the Identification, Evaluation, and Treatment of Overweight and Obesity in Adults. Executive summary of the clinical guidelines on the identification, evaluation, and treatment of overweight and obesity in adults. Arch Intern Med 1998;158:1855-67.

[4] Perlstein ResMI,Bissada NF(1977) Influence of obesity and hypertension on the severity of periodontitis in rats.Oral Surg Med Oral Pathol 43;707-719.

[5] Saito. Shimazaki Y,Koga T,et al (2001).Relationship between upperbody obesity and Periodontitis. J Dent Res 80:1631-1636.

[6] Pouliot MC, Despreés JP, Lemieux S, Moorjani S, Bouchard C, Tremblay A, et al. Waist circumference and abdominal sagittal diameter: Best simple anthropometric indexes of abdominal visceral adipose tissue accumulation and related cardiovascular risk in men and women. Am J Cardiol 1994;73:460-8.

[7] Wang Y, Rimm EB, Stampfer MJ, Willett WC, Hu FB. Comparison of abdominal adiposity and overall obesity in predicting risk of type 2 diabetes among men.AmJClinNutr2005;81:555-63.

[8] Yusuf S, Hawken S, Ounpuu S, Bautista L, Franzosi MG, Commerford P, et al. Obesity and the risk of myocardial infarction in 27,000 participants from 52 countries:Acase-controlstudy.Lancet2005;366:1640-9.

[9] Ritchie CS. Obesity and periodontal disease. Periodontol 2000 2007;44:154-63

[10] Pischon N, Heng N, Bernimoulin JP, Kleber BM, Willich SN, Pischon T. Obesity, inflammation and periodontal disease. J Dent Res 2007;86:400-9.

[11] Nishida N, Tanaka M, Hayashi N, Nagata H, Takeshita T, Nakayama K, et al. Determination of smoking and obesity as periodontitis risks using the classification and regression tree method. J Periodontol.2005;76:923-8.

[12] Gidding SS, Dennison BA, Birch LL., et al. Dietary recommendations for children and adolescents. A guide for practitioners. Consensus Statement from the American Heart Association.Circulation2005;112:2061-2075. GencoRJ,GrossiSG,HoA,NishimuraF,MurayamaY(2005).AProposedmodellinking inflammationtoobesity,diabetes and periodontal infections.J Periodontol 76(11 suppl):2075-2084.

[13] LoescheW,KarapetowF,PohlAetal. Plasma lipid and blood glucose levels in patients with destructiveperiodontaldisease JClinPeriodontol2000;27:537-541.

[14] BeckJD,Pankowetal;Dentalinfectionandatherosclerosis;AmHeartJ1999;48:528-33.

[15] BarbaraNoack,Ingeborg Jachmann, et al :Metabolic diseases and their possible link to risk indicators of periodontitis. J Periodontol 2000;71;898-903.

[16] Christopher W.Cutler, Edward A Shinedling,et al.Association between periodontitis and hyperlipidemia; Cause or effect. J Periodontol 1999; 12:1429-1434. 